\title{
Synchronization circuit with sidelobe cancellation
}

\author{
Young-Joon Song ${ }^{\text {a) }}$ and In-Sik Kang \\ Department of Electronic Engineering, Kumoh National Institute of Technology, 1 \\ Yangho-dong, Gumi, Gyungbuk, 730-701, Korea \\ a)yjsong@kumoh.ac.kr
}

\begin{abstract}
Synchronization can be achieved by computing the correlation of a received signal using a locally stored synchronization sequence. The segment giving the maximum correlation is taken as the location of the sequence. In this letter, we present an ideal synchronization circuit using a sequence pair with bipolar correlation. When a sequence pair is inputted, the circuit outputs double maximum correlation values that are equal in magnitude and opposite in polarity at the zero and middle shifts, by using the sidelobe cancellation effect between the two sequences. Such a circuit is particularly useful for synchronization, since it can be used to double-check the synchronization timing.
\end{abstract}

Keywords: synchronization, sidelobe cancellation, bipolar correlation

Classification: Wireless circuits and devices

\section{References}

[1] R. A. Scholtz, "Frame synchronization techniques," IEEE Trans. Commun., vol. 28, no. 4, pp. 1204-1213, Aug. 1980.

[2] M. Simon, J. Omura, R. Scholtz, and B. Levitt, Spread Spectrum Communications Handbook, McGraw-Hill, 1994.

[3] Y. J. Song, S. L. Kwon, Y. S. Moon, and H. S. Shin, "Frame synchronization confirmation technique using pilot pattern in W-CDMA System," APCC/OECC'99, vol. 1, pp. 662-665, Oct. 1999.

[4] Y. J. Song, "Frame synchronization confirmation technique using pilot pattern," J. Communications and Networks, vol. 2, no. 1, pp. 69-73, March 2000.

[5] Y. J. Song, "Frame synchronization word in W-CDMA system," IEEE Commun. Lett., vol. 7, no. 11, pp. 520-522, Nov. 2003.

[6] J. Wolfman, "Almost perfect autocorrelation sequences," IEEE Trans. Inf. Theory, vol. 38, no. 4, pp. 1412-1418, 1992.

[7] D. Chu, "Polyphase codes with good periodic correlation properties," IEEE Trans. Inf. Theory, pp. 531-532, July 1972.

[8] M. Golay, "Complementary series," IRE Trans. Inf. Theory, vol. IT-11, pp. 82-87, April 1961.

[9] S. Budisin, "Efficient pulse compressor for Golay complementary sequences," Electronics Letters, vol. 27, no. 3, pp. 219-220, Jan. 1991.

[10] B. Popovic, "Efficient Golay correlator," Electronics Letters, vol. 35, no. 17, pp. 1427-1428, Aug. 1999. 


\section{Introduction}

Synchronization sequences with special correlation properties can be inserted periodically into a bit stream to check the time of the transmitter and receiver by using correlations $[1,2,3,4,5]$. The sequences usually have zero or extremely low out-of-phase correlation values for optimum performance $[5,6]$. If the out-of-phase auto-correlation coefficients of a sequence are all zero, the sequence is said to have perfect autocorrelation. However $(0,1,1,1)$ is the only binary sequence with perfect autocorrelation [6]. Chu proposed polyphase codes with perfect autocorrelation [7], but when taking hardware complexity into consideration, binary codes are preferred. Golay proposed pairs of complementary binary sequences, which have the sum of their autocorrelation functions equal to zero for all time shifts except zero [8]. Because of their excellent correlation properties, as well as the efficient hardware implementation of Golay complementary sequences of length $2^{N}$, Golay codes are widely used in applications in many fields, such as synchronization in wireless communication systems [9, 10].

In this letter we present an ideal synchronization circuit, which outputs double maximum correlation values that are equal in magnitude and opposite in polarity at the zero and middle shifts. On the transmitter side, a set of complementary sequences with the sum of their autocorrelation functions equal to zero for all time shifts (except the zero and middle shifts) is assigned to a pair of I and Q channels of a quadrature phase shift keying (QPSK) modulator. Assuming that the channel is distortionless, the output of the receiver has an ideal bipolar correlation function owing to the sidelobe cancellation effect. The addition of the two correlation functions does not affect the synchronization process. Thus, we can achieve the same effect as that obtained when using a perfect autocorrelation sequence. This circuit is particularly useful for synchronization, as it can be used to double-check the synchronization timing and thus to reduce the synchronization search time. Computer searches are carried out to find complementary sequence pairs with ideal bipolar correlation properties.

\section{Properties of autocorrelation}

Let $s=\left\{s_{i}\right\}$ be a binary sequence of period $n$. If the components of $s$ are cyclically shifted one place to the left, the resulting sequence is $\boldsymbol{s}^{(1)}=$ $\left(s_{1}, s_{2}, \cdots, s_{0}\right)$. For two integers, $i$ and $j, \boldsymbol{s}^{(i)}=\boldsymbol{s}^{(j)}$ if $i \equiv j(\bmod n)$. The periodic autocorrelation function is defined by

$$
R(\tau)=\sum_{t=0}^{n-1}(-1)^{s_{t}+s_{(t+\tau)} \bmod n}
$$

where $\bmod n$ denotes modulo $n$, and $s_{t}+s_{(t+\tau) \bmod n}$ is computed as modulo 2 . $R(0)$ is known as the in-phase autocorrelation value, whereas $R(\tau)$ for $(\tau \neq 0)$ are known as out-of-phase auto-correlation values. The out-of-phase autocorrelation values are also called sidelobes. If the out-of-phase autocorrelation coefficients of a sequence are all zero, the sequence $s$ is called a perfect autocorrelation sequence. However $(0,1,1,1)$ is the only binary sequence with 
perfect autocorrelation. Now, let $\|s\|$ denote the number of "one" values in $s$. Then, the number of like digits between the original sequence and a sequence shifted by $\tau$ units is $n-2(\|s\|-m)$, where $m$ is the number of "one" elements coincident between $s$ and $\boldsymbol{s}^{(\tau)}$. Likewise, the number of unlike digits between $s$ and $s^{(\tau)}$ is $2(\|s\|-m)$. Hence, the autocorrelation function becomes

$$
R(\tau)=n-4(\|s\|-m) .
$$

A sequence pair, $\left(s_{1}, s_{2}\right)$, is called a complementary sequence pair with ideal bipolar correlation if it satisfies

$$
R_{1}(\tau)+R_{2}(\tau)=\left\{\begin{array}{c}
2 n, \quad \tau=0 \\
-2 n, \quad \tau=n / 2 \\
0, \text { elsewhere }
\end{array}\right.
$$

\section{Sequence pairs}

From Equation (2), we can see that binary sequences with periods of a multiple of 4 can have zero out-of-phase coefficients. Therefore, in this letter we consider sequences with periods that are multiples of 4 . However, $(0,1,1,1)$ is the only perfect autocorrelation sequence, and $(0,0,1,1)$ is the only sequence

Table I. Complementary sequence pairs with ideal bipolar correlation properties.

\begin{tabular}{|c|c|}
\hline Pair number & Sequence pairs \\
\hline $8 \mathrm{~A}$ & 00001111 \\
$8 \mathrm{~B}$ & 01001011 \\
\hline $12 \mathrm{~A}$ & 010000101111 \\
$12 \mathrm{~B}$ & 001000110111 \\
\hline $16 \mathrm{~A}$ & 0010000011011111 \\
$16 \mathrm{~B}$ & 0010100011010111 \\
\hline $16 \mathrm{C}$ & 0001000011101111 \\
$16 \mathrm{D}$ & 0100100010110111 \\
\hline $20 \mathrm{~A}$ & 00101000001101011111 \\
\hline $20 \mathrm{~B}$ & 00010010001110110111 \\
\hline $20 \mathrm{C}$ & 01100100001001101111 \\
\hline $20 \mathrm{D}$ & 00010100001110101111 \\
\hline $24 \mathrm{~A}$ & 01010100000110001011111 \\
\hline $24 \mathrm{~B}$ & 00110101000000001010011011111 \\
\hline $28 \mathrm{~A}$ & 001010011000001011010110011111 \\
\hline $28 \mathrm{~B}$ & 0010101000000011010101111111 \\
\hline $28 \mathrm{C}$ & 0001101101100011100100100111 \\
\hline $28 \mathrm{D}$ & 0001100101000000111001101011111 \\
\hline $32 \mathrm{~A}$ & 0011011010100000110010010101111 \\
\hline $32 \mathrm{~B}$ & 00101011000000001101010011111111 \\
\hline $32 \mathrm{C}$ & 01001100011010001011001110010111 \\
\hline $32 \mathrm{D}$ & 000010011000100000111101100111011111 \\
\hline $36 \mathrm{~A}$ & 001101001010100000110010110101011111 \\
\hline $36 \mathrm{~B}$ & 000101011000100000111010100111011111 \\
\hline $36 \mathrm{C}$ & 001110110110100000110001001001011111 \\
\hline $36 \mathrm{D}$ & 0110110111010001000010010010001011101111 \\
\hline $40 \mathrm{~A}$ & 0100001100011101000010111100111000101111 \\
\hline $40 \mathrm{~B}$ & 0100111101110001000010110000100011101111 \\
\hline $40 \mathrm{C}$ & \\
\hline $40 \mathrm{D}$ & 0010111011011101000011010001001000101111 \\
\hline
\end{tabular}


with an ideal bipolar correlation function. Table I shows the complementary sequence pairs of periods of $4 k$ for $k=2, \cdots, 10$. Computer searches were carried out to find the sequence pairs with bipolar correlations. The autocorrelation functions have two peak values at the zero and middle shifts with non-zero out-of-phase coefficients. Because of the sidelobe cancellation, except at the zero and middle shifts of the autocorrelation functions of two sequences in the same pair, after combination of the two functions, an ideal bipolar correlation function can be realized. The ideal bipolar correlation property is particularly useful for synchronization, since it can be used to double-check the synchronization timing.

\section{Synchronization circuit}

Figure 1 shows the transmitter and receiver for synchronization using a complementary sequence pair with bipolar correlation. On the transmitter side, a complementary sequence pair $(\boldsymbol{a}, \boldsymbol{b})$ is assigned to a pair of I and Q channels of a QPSK modulator, as depicted in Figure 1(a). On the side of the receiver, the pair of I and $\mathrm{Q}$ channels correlates the received signal with $\boldsymbol{a}$ and $\boldsymbol{b}$, as shown in Figure 1 (b). Assuming that the channel is distortionless, as depicted in Figure 1 (c), the output of the receiver shows an ideal bipolar correlation function except at the middle shift, because of the sidelobe cancellation effect. The addition of the two correlation functions does not affect the synchronization process, and the two peak values of the autocorrelation function at the zero and middle shifts are enhanced by $\pm 2 n$. Thus, by setting the double thresholds to the correlation values at the zero and middle shifts as shown in Figure 1(b), we can achieve the same effect as that obtained using an ideal synchronization sequence and further reduce the synchronization search time since it can double-check the synchronization timing. The hardware increase to implement the proposed device compared to that of using Goly codes is double thresholds detector whose complexity increase is negligible. If the length of the synchronization sequence is $2^{N}$, the hardware improvement factor using Golay codes is proportional to $2^{N} / N$ because of the excellent correlation properties of Golay codes of $2^{N}[9]$.

\section{Synchronization time performance}

Synchronization sequences with special correlation properties can be inserted into a bit stream to align the timing of the transmitter and receiver by using correlations. If the computed correlation value is smaller than a predefined threshold value, the receiver-stored synchronization sequence is shifted by one bit, and another autocorrelation operation is processed for the next time period $T$. This process is repeated until a "hit" (output above the threshold) is observed by the threshold detector. This process is equivalent to code acquisition. If the "hit" is true, the receiver has acquired synchronization and the search is complete. If the "hit" is not true (a false alarm), the receiver fails the synchronization and retries the code acquisition.

Chu proposed polyphase codes with perfect autocorrelation [7], and Golay 


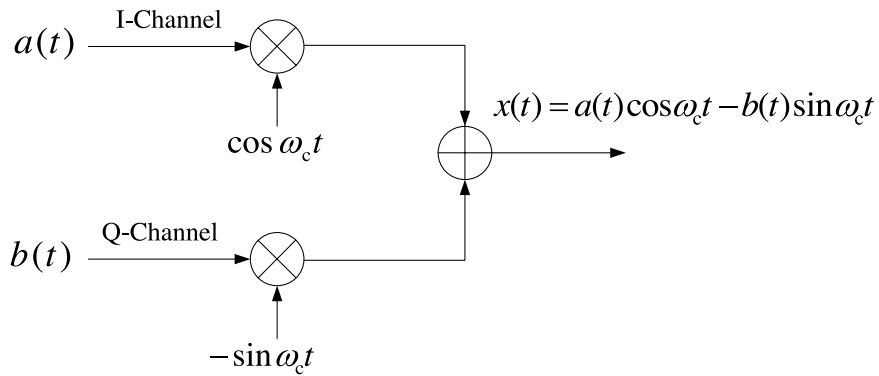

(a)

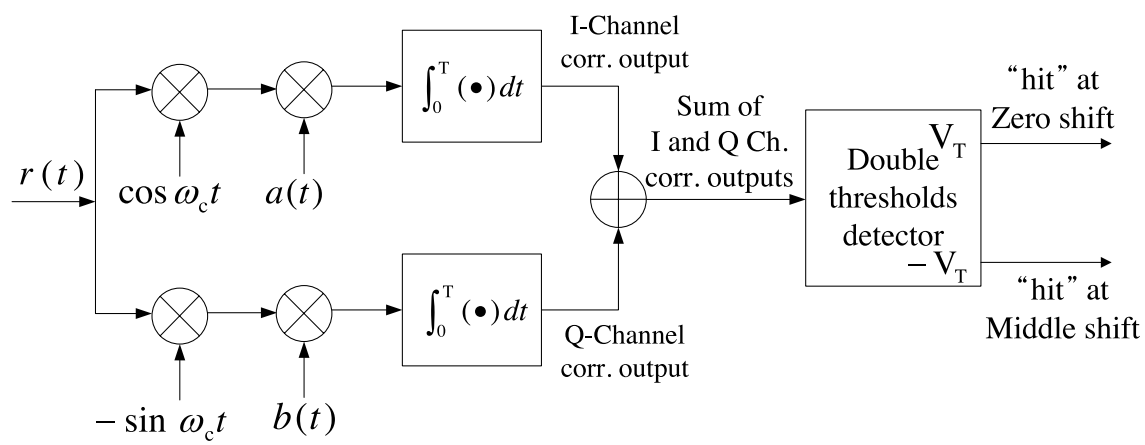

(b)
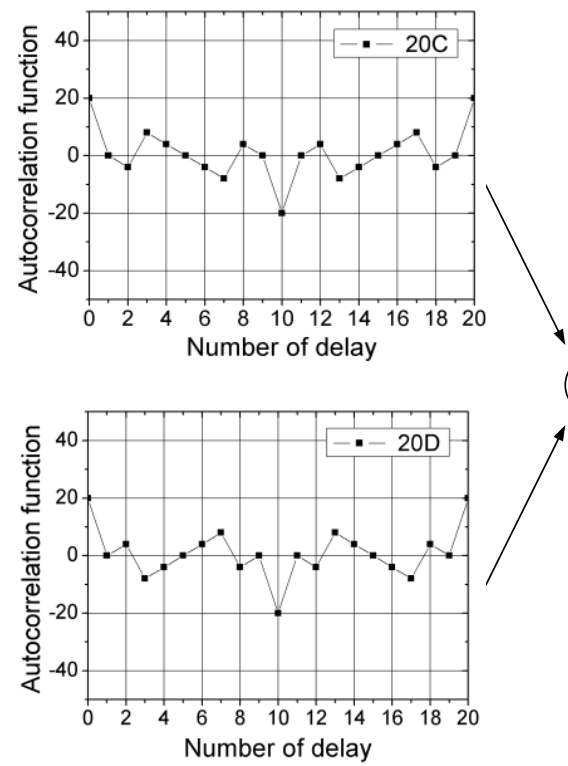

(2)

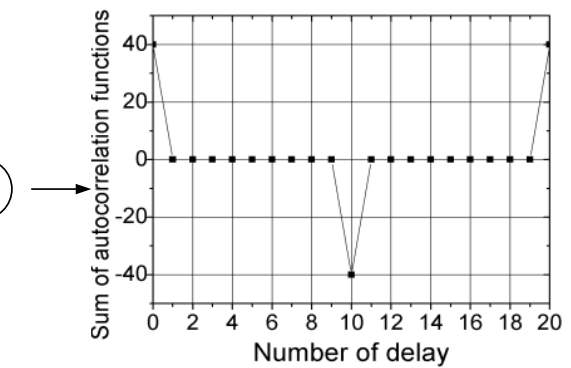

(c)

Fig. 1. (a) Transmitter, (b) receiver, and (c) sum of corr. outputs for synchronization using a complementary sequence pair with ideal bipolar correlation.

proposed pairs of complementary binary sequences that have the sum of their autocorrelation functions equal to zero for all time shifts except zero [8]. To compare the synchronization time performance between the proposed code and conventional codes, we carried out intensive simulations and the results are shown in Figure 2 where the Chu polyphase code $\left\{a_{k}=\exp \left(i \frac{\pi k^{2}}{16}\right), k=\right.$ $0,1, \cdots, 15\}$, the Golay codes (1000110110000010,0100000101001110), and 


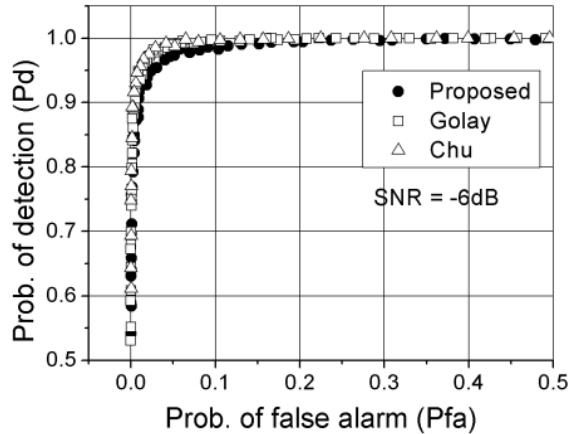

(a)

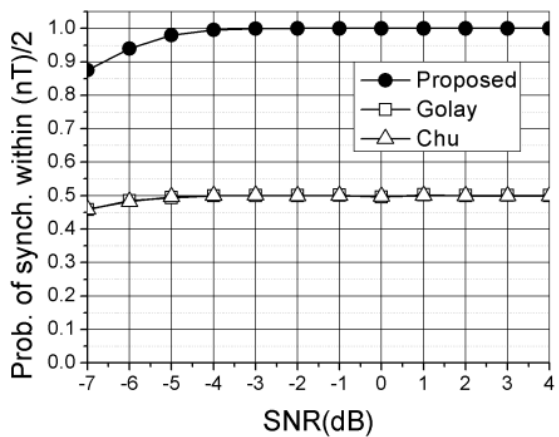

(b)

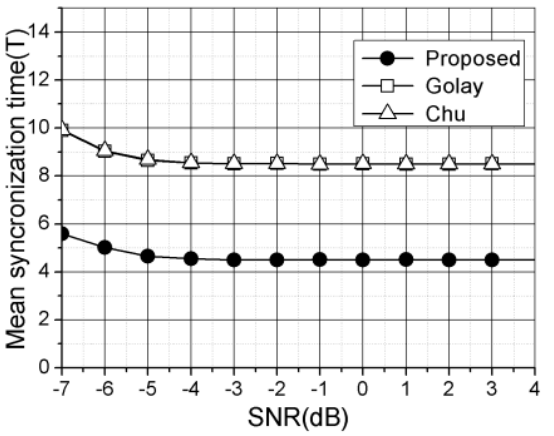

(c)

Fig. 2. (a) ROC curves, (b) probability that synchronization time is within $(n T) / 2$, and (c) mean synchronization time performance.

the proposed codes (16 A, 16B) of Table I are used. Figure 2 (a) shows receiver operating characteristic $(\mathrm{ROC})$ curves at $\mathrm{SNR}=-6 \mathrm{~dB}$ for the proposed code and the conventional codes. From the curves, we see that all codes have the excellent probability of false alarm $\left(P_{f a}\right)$ and probability of detection $\left(P_{d}\right)$. At the higher SNR values, we obtain the better ROC curves. Figure 2 (b) shows the probability that a receiver can achieve the synchronization within $(n T) / 2$. It shows that the proposed method has two times higher probability of synchronization than that of using the conventional codes because of the ideal bipolar correlation property of the proposed codes, which enables us to check the synchronization timing at zero and middle shifts. This is confirmed in Figure 2 (c) which compares the mean synchronization time between the 
proposed code and the conventional codes. In this simulation, the threshold values for the proposed and conventional codes are set to have $P_{f a}=0.015$. The channel used in this simulation is additive white Gaussian noise (AWGN) and the receiver assumes the coherent detection. The proposed method is approximately twice as good as that using the conventional codes for both high and low signal-to-noise ratios (SNRs) of AWGN channel. Because of the excellent correlation properties of Golay codes of $2^{N}$, the hardware improvement factor using Golay codes is proportional to $2^{N} / N$ [9]. Thus, if the length of the synchronization sequence is $2^{N}$ and the hardware complexity is more important than the synchronization performance, a synchronization circuit using Golay codes is preferred. Otherwise, the proposed synchronization circuit can be used, since it can double-check the synchronization timing and thus reduce the synchronization search time.

\section{Conclusion}

We have proposed an efficient synchronization circuit using complementary sequence pairs with ideal bipolar correlation properties. Using the proposed correlation circuit, which employs the sidelobe cancellation between two correlation values from the I and Q channels, we can obtain ideal synchronization, which outputs two maximum values equal in magnitude and opposite in polarity at the zero and middle shifts. We compared the synchronization time performance of the proposed device with those of conventional methods in a noisy channel environment. We found that the method using the proposed code is approximately twice as good as that using the conventional codes. Thus, the proposed synchronization device can reduce the synchronization search time, since it can double-check the synchronization timing.

\section{Acknowledgments}

This paper was supported by Research Fund, Kumoh National Institute of Technology. 